\title{
Bilevel Optimal Control, Equilibrium, and Combinatorial Problems with Applications to Engineering
}

\author{
Vyacheslav Kalashnikov, ${ }^{1,2,3}$ Stephan Dempe, ${ }^{4}$ Boris Mordukhovich, ${ }^{5}$ and Sergii V. Kavun ${ }^{6}$ \\ ${ }^{1}$ Department of Systems \& Industrial Engineering, Tecnológico de Monterrey (ITESM), Campus Monterrey, \\ Ave. Eugenio Garza Sada 2501 Sur, Monterrey, NL 64849, Mexico \\ ${ }^{2}$ Department of Experimental Economics, Central Economics \& Mathematics Institute (CEMI), Russian Academy of Sciences, \\ Nakhimovsky Pr. 47, Moscow 117418, Russia \\ ${ }^{3}$ Department of Computer Science, Sumy State University, Rimsky-Korsakov St. 2, Sumy 40007, Ukraine \\ ${ }^{4}$ Department of Optimization, Institute for Numerical Mathematics and Optimization, TU Bergakademie Freiberg, \\ 09596 Freiberg, Germany \\ ${ }^{5}$ Department of Mathematics, Wayne State University, 656 West Kirby Avenue Detroit, MI 48202, USA \\ ${ }^{6}$ Kharkiv Institute of Banking, University of Banking of the National Bank of Ukraine, Peremogy Avenue 55, Kharkiv 61174, Ukraine \\ Correspondence should be addressed to Vyacheslav Kalashnikov; kalash@itesm.mx
}

Received 2 November 2017; Accepted 5 November 2017; Published 14 December 2017

Copyright (C) 2017 Vyacheslav Kalashnikov et al. This is an open access article distributed under the Creative Commons Attribution License, which permits unrestricted use, distribution, and reproduction in any medium, provided the original work is properly cited.

In recent years, a large literature on bilevel programming in finite-dimensional spaces has emerged, much of it focusing on optimality conditions, theory, and algorithms associated with certain classes of such problems. The topic proposed to treat in this special issue goes one step further in attempting to deal with bilevel programming problems extended to infinite-dimensional spaces at the upper level, at the lower level, and at both. Such classes of optimal control problems often arise in engineering applications: robotics, water, and waste control problems, ecology in various environments, and so on.

Bilevel Optimal Control Problems are a special class of optimization problems combining Bilevel Programming Programs and optimal control theory. These problems are a generalization of the optimal control problems in cases with more than one decision maker. However, this new type of problems is much more complicated than optimal control problems in the sense that what constitutes a solution, for example, in the particular case of optimistic/pessimistic Stackelberg differential games, is no longer obvious. A lot of new ideas and approaches have been developed in this area by the active researchers throughout the world; see, for example, [1-6], among other publications.
A general formulation for Bilevel Optimal Control Problems includes an optimal control problem in both levels, commonly linked with the dynamical system. Nevertheless, other less investigated problems in this field of research can be treated. Hence, this special issue also invites contributions dealing with a special class of optimistic Bilevel Optimal Control Problems where only the upper level possesses an optimal control problem whereas the lower level is a finitedimensional problem whose parameter is the final state of the upper level state function. Recall that the optimistic approach models a cooperative behavior of leader and follower while the pessimistic one depicts a competitive situation. Thus, various optimality conditions can be derived by replacing the lower level problem by either its primal-dual optimality conditions or its optimal value function.

Although a wide range of applications fit the bilevel optimal control framework, real-life implementations are scarce, due mainly to the lack of efficient algorithms for tackling medium- and large-scale bilevel programming problems to which the Bilevel Optimal Control Problems are often reduced. Solving a bilevel (more generally, hierarchical) optimization problem, even in its simplest form, is a difficult task. A lot of different alternative methods may be used based 
on the structure of the problem analyzed, but there is no general method that guarantees convergence, performance, or optimality for every type of problem.

Mixed-integer bilevel programming problems (with part of variables at the upper and/or lower level being integer/Boolean ones) are even harder for the well-known conventional optimization techniques. For instance, a usual replacement of the lower level optimization problem with a corresponding KKT condition may not work if some lower level variables are not continuous. Therefore, the solid theoretical base including elements of combinatorial methods is necessary to be found, in order to propose efficient algorithmic procedures aimed at finding local or global solutions of such a problem.

Last but not least many new applied problems in the area of energy networks have recently arisen that can be efficiently solved only as mixed-integer bilevel optimal control programs. Among them are the natural gas cash-out problem, the deregulated electricity market equilibrium problem, biofuel problems, a problem of designing coupled energy carrier networks, wastewater control problems, and so on, if we mention only part of such applications. Bilevel models to describe migration processes are also in the running of the most popular new themes in the area of bilevel programming.

Engineering applications of bilevel optimization and combinatorial problems also include facility location, environmental regulation, energy and agricultural policies, hazardous materials management, and optimal designs for chemical and biotechnological processes.

This special volume comprises papers dealing with three main topics: bilevel programming and optimal control, equilibrium models, and combinatorial (integer programming) problems and their applications to engineering.

The volume opens with the research paper "Defense Strategy of Aircraft Confronted with IR Guided Missile" by $\mathrm{H}$. Huang et al. that proposes and studies a model of aircraft and a surface-type infrared (IR) decoy. The latter can simulate the IR characteristics of the target aircraft, hence being one of the most effective equipment to confront IR guided missile. The authors investigate into the aircraft maneuver and radiation models based on real data of flight and exhaust system radiation in the state of different heights and different speeds. Thus, the most effective avoidance maneuver is simulated when the missile comes from the front of the target aircraft. Lastly, combining maneuvers with decoys, the best defense strategy is analyzed when the missile comes from the front of the aircraft. The result of the simulation, which is authentic, is propitious to avoid the missile and improve the survivability of the aircraft.

The next paper "Capacity Allocation and Revenue Sharing in Airline Alliances: A Combinatorial Auction-Based Modeling" by Y.-J. Gu and J.-F. Zhu attempts to establish a framework to help airline alliances effectively allocate their seat capacity with the aim of maximizing the alliances' revenue. By assuming the airline alliance to behave as an auctioneer with the seat capacity in an itinerary as lots, the Combinatorial Auction Model is constructed to optimize the allocation of the seat. To this end, the authors propose the revenue sharing method sharing revenue among the partners with the aid of the Vickrey-Clarke-Groves (VCG) tools. The results of the numerical study show that the seat capacity allocation is effective even without the complete information exchange, and the twofold revenue share method is more efficient for the airlines.

The contribution titled "Embodied Energy and Cost Optimization of RC Beam under Blast Load" was proposed by $\mathrm{R}$. Yu et al. The authors note that reinforced concrete (RC) structures not only consume a lot of resources but also cause enormous continuing pollution. However, sustainable design of beams could make the RC structures more environment-friendly. One of the important indices for environmental impact assessment is embodied energy. The aim of the presented study is to optimize the embodied energy and the cost of RC beam subject to the blast loads. First, a general optimization procedure is described. Then, this procedure is used to optimize the embodied energy and the costs of RC beams. The optimal results for the costs and the embodied energy are then compared. It is demonstrated that the optimization results are strongly affected by the cost ratio $n_{C}$ (the ratio of the steel price to the price of concrete per unit volume) and the embodied energy ratio $n_{E}$ (the ratio of the embodied energy of steel to the embodied energy of concrete per unit volume). An optimal design that minimizes both the embodied energy and the cost simultaneously is obtained whenever the values of $n_{C}$ and $n_{E}$ are close enough.

An interesting piece of research titled "Multidimensional Dynamic Programming Algorithm for N-Level Batching with Hierarchical Clustering Structure" has been presented by J.-G. Kim et al. Their study focuses on the $N$-level batching problem with a hierarchical clustering structure. Clustering is the task of grouping a set of item types in such a way that the item types in the same cluster, in certain sense, are closer to each other than to those in other clusters. In a hierarchical clustering structure, more and more different item types are clustered together while the level of the hierarchy increases. The $N$-level batching is the process by which the items of different types are grouped into several batches passing from level 1 to level $N$ sequentially. This batching is completed for a given hierarchical clustering structure such that the batches in each level satisfy the maximum and minimum batch size requirements of the level. The authors of the paper consider two types of processing costs of the batches: the unit processing cost and the batch processing cost. Then they formulate the $N$-level batching problem with a hierarchical clustering structure as a nonlinear integer programming model with the objective of minimizing the total processing cost. To solve the optimization problem, the authors propose a multidimensional dynamic programming algorithm illustrated with an example.

An important practical problem is addressed by Q. Liu et al. in their study "Stability Analysis of Water-Resistant Strata in Karst Tunnel Based on Releasable Elastic Strain Energy." In this paper, the energy instability criterion of water-resistant strata and rock mass failure index (RMFI) are proposed based on releasable elastic strain energy $U^{e}$. The 
RMFI is employed to represent the damage extent of waterresistant strata. When $\mathrm{RMFI}<1.0$, the rock mass is stable, and if $\mathrm{RMFI}=1.0$, the rock mass is in the critical instability state. However, if RMFI $>1.0$, the rock mass is unstable. A numerical procedure to determine the releasable elastic strain energy $U^{e}$ and the index RMFI is proposed and performed with the aid of the FISH programming language of the Flac ${ }^{3 \mathrm{D}}$ software. Afterward, the authors apply $\mathrm{Flac}^{3 \mathrm{D}}$ in order to analyze the distribution law of the releasable elastic strain energy $U^{e}$ and the form of a failure zone for different values of the width of a concealed karst cave. Finally, combined with the numerical analysis, a case study is carried out to illustrate the proposed technique's rationality, effectiveness, and feasibility of the use of RMFI for predicting the safe thickness of the water-resistant strata.

The paper "Data Analytics Based Dual-Optimized Adaptive Model Predictive Control for the Power Plant Boiler" by $Z$. Tang et al. deals with the control of the furnace temperature of a power plant boiler. In order to do that with a high level of precision, a dual-optimized adaptive model predictive control (DoAMPC) method is designed based on the data analytics. In the proposed DoAMPC, an accurate predictive model is constructed adaptively by the hybrid algorithm of the least squares support vector machine and differential evolution method. Then, an optimization problem is constructed based on the predictive model with many constraints. To control the boiler furnace temperature, the differential evolution method is utilized, which finds the best values of the control variables by solving the optimization problem in question. The proposed algorithm can adapt to the time-varying situation by updating the sample data. The experimental results based on practical data illustrate that the DoAMPC can control the boiler furnace temperature with errors less than $1.5 \%$, which can readily meet the requirements of the real production process.

The volume is finished with the paper "A New Calculation Method of Dynamic Kill Fluid Density Variation during Deep Water Drilling" proposed by H. Fan with coauthors. It is wellknown that there are plenty of uncertainties and enormous challenges in deep water drilling due to complicated shallow flows and deep strata of high temperature and pressure. This paper investigates density of a dynamic kill fluid and the optimum density during the kill operation process, in which the dynamic kill process can be divided into two stages, namely, the dynamic stable stage and the static stable stage. The dynamic kill fluid consists of a single liquid phase and different solid phases. In addition, the liquid phase is a mixture of water and oil. Therefore, a new method for calculating the temperature and pressure field of deep water wellbore is proposed. The paper calculates the changing trend of the kill fluid density under different temperature and pressure values by means of a superposition method, a nonlinear regression, and a segment processing technique. By employing the improved model of kill fluid density, deep water kill operation in a well is investigated. A comparison shows that the calculated density results are in line with the field data. The model proposed in this paper proves to be satisfactory in optimizing the dynamic kill operations ensuring the safety in the deep water.

\section{Acknowledgments}

Our enormous gratitude is due to the long list of referees, whose valuable comments and suggestions have helped us a lot in making our final decisions about accepting the papers for publication in the journal.

Vyacheslav Kalashnikov Stephan Dempe Boris Mordukhovich Sergii V. Kavun

\section{References}

[1] S. Dempe, B. S. Mordukhovich, and A. B. Zemkoho, "Necessary optimality conditions in pessimistic bilevel programming," Optimization. A Journal of Mathematical Programming and Operations Research, vol. 63, no. 4, pp. 505-533, 2014.

[2] S. Dempe and J. Dutta, "Is bilevel programming a special case of a mathematical program with complementarity constraints?" Mathematical Programming, vol. 131, no. 1-2, Ser. A, pp. 37-48, 2012.

[3] D. Catanzaro, E. Gourdin, M. Labbé, and F. A. Özsoy, "A branchand-cut algorithm for the partitioning-hub location-routing problem," Computers \& Operations Research, vol. 38, no. 2, pp. 539-549, 2011.

[4] G. Heilporn, M. Labbé, P. Marcotte, and G. Savard, "A polyhedral study of the network pricing problem with connected toll Arcs," Networks, vol. 55, no. 3, pp. 234-246, 2010.

[5] S. T. DeNegre and T. K. Ralph, "A branch-and-cut algorithm for integer bilevel linear programs," in Operations Research and Cyber-Infrastructure, vol. 47 of Series Research/Computer Science Interfaces, pp. 65-78, Springer, 2009.

[6] L. Liberti and C. C. Pantelides, "An exact reformulation algorithm for large nonconvex NLPs involving bilinear terms," Journal of Global Optimization, vol. 36, no. 2, pp. 161-189, 2006. 


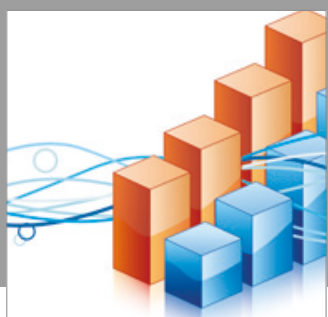

Advances in

Operations Research

vatersals

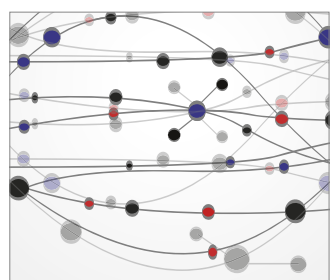

\section{The Scientific} World Journal
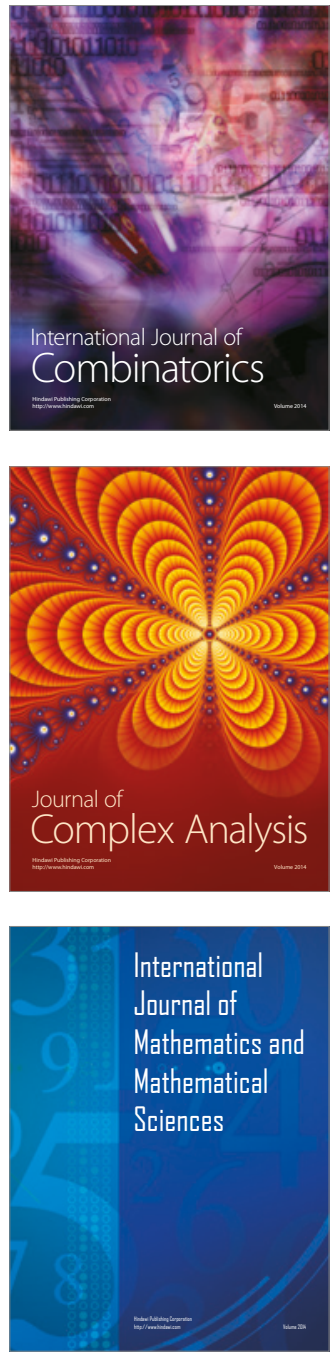
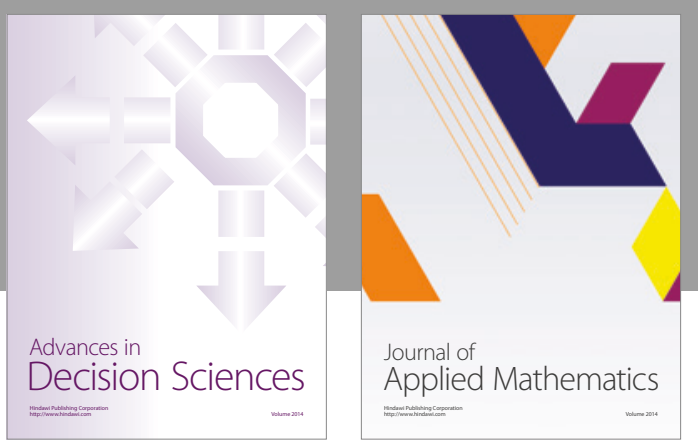

Algebra

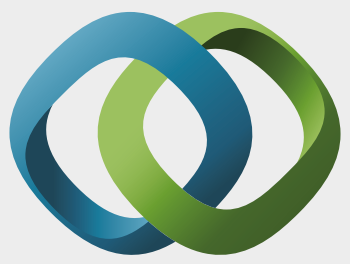

\section{Hindawi}

Submit your manuscripts at

https://www.hindawi.com
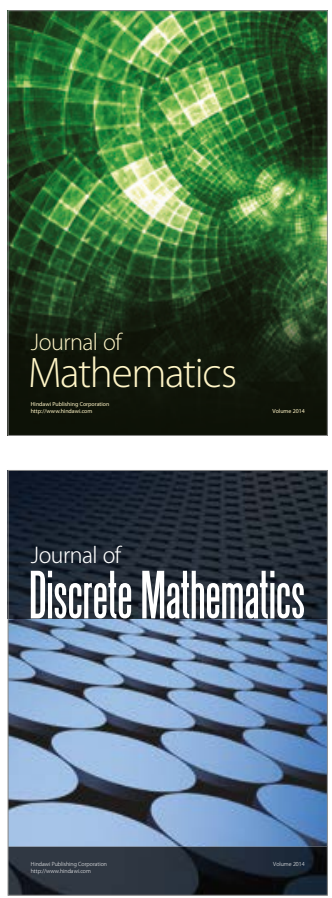

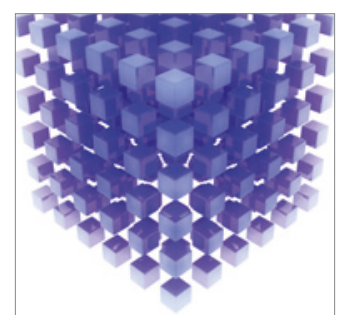

Mathematical Problems in Engineering
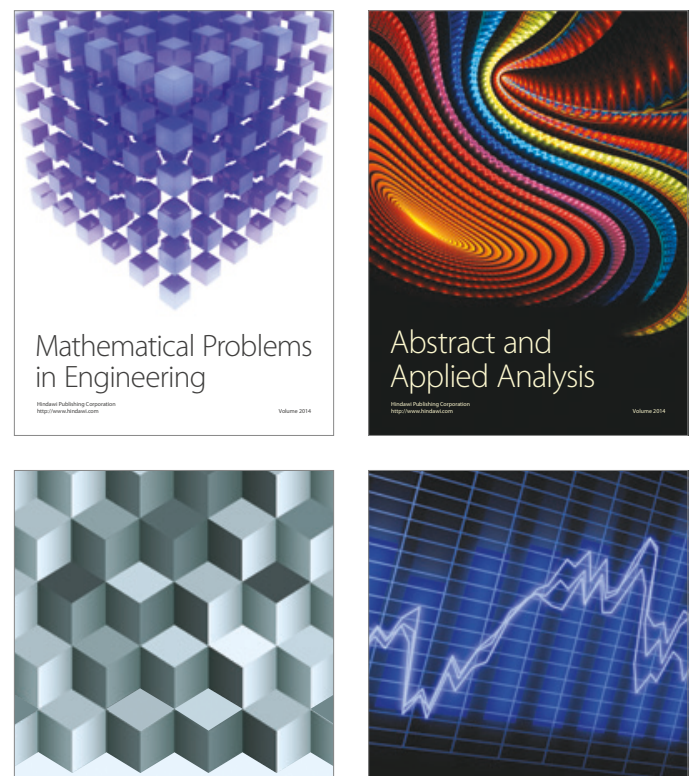

Journal of

Function Spaces

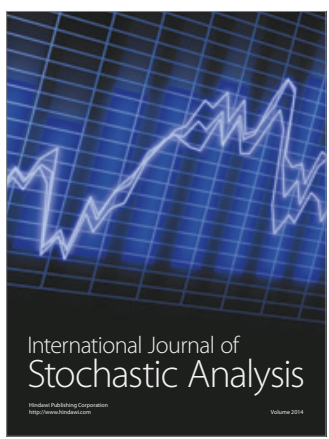

Probability and Statistics
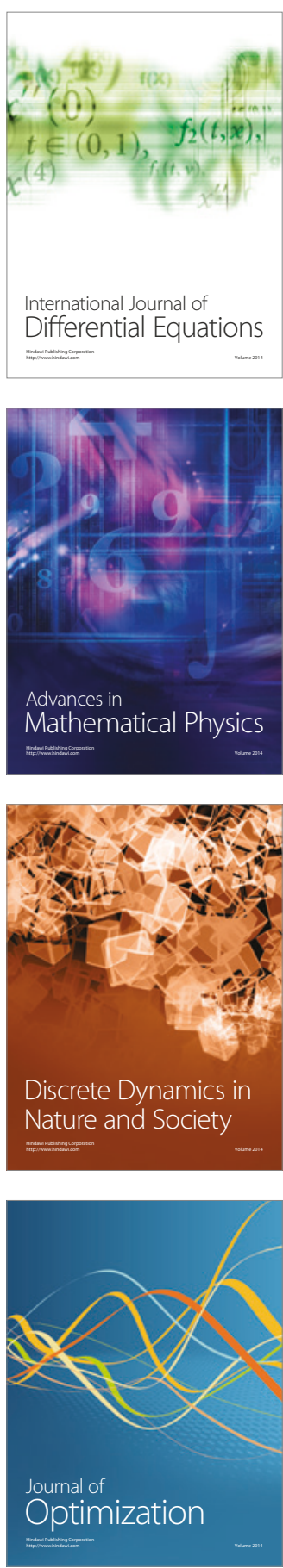